\title{
Aggressive prolactinoma (Review)
}

\author{
ANA VALEA $^{1,2}$, FLORICA SANDRU ${ }^{3,4}$, AIDA PETCA ${ }^{5,6}$, MIHAI CRISTIAN DUMITRASCU ${ }^{5,7}$, \\ MARA CARSOTE $^{8,9}$, RAZVAN-COSMIN PETCA ${ }^{10,11}$ and ADINA GHEMIGIAN ${ }^{8,9}$
}

\author{
${ }^{1}$ Department of Endocrinology, 'I. Hatieganu' University of Medicine and Pharmacy, 400012 Cluj-Napoca; \\ ${ }^{2}$ Department of Endocrinology, Clinical County Hospital, 400000 Cluj-Napoca; ${ }^{3}$ Department of Dermatology, \\ 'Carol Davila' University of Medicine and Pharmacy, 050474 Bucharest; ${ }^{4}$ Department of Dermatology, \\ 'Elias' University Emergency Hospital, 011461 Bucharest; ${ }^{5}$ Department of Obstetrics and Gynecology, \\ 'Carol Davila' University of Medicine and Pharmacy, 020021 Bucharest; ${ }^{6}$ Department of Obstetrics and Gynecology, \\ 'Elias' University Emergency Hospital, 011461 Bucharest; ${ }^{7}$ Department of Obstetrics and Gynecology, \\ University Emergency Hospital, 050098 Bucharest; ${ }^{8}$ Department of Endocrinology, 'Carol Davila' University of \\ Medicine and Pharmacy, 050474 Bucharest; ${ }^{9}$ Department of Endocrinology, 'C. I. Parhon' National Institute of \\ Endocrinology, 011863 Bucharest; ${ }^{10}$ Department of Urology, 'Carol Davila’ University of Medicine and Pharmacy, \\ 050474 Bucharest; ${ }^{11}$ Department of Urology, 'Prof. Dr. Theodor Burgele' Clinical Hospital, 061344 Bucharest, Romania
}

Received July 6, 2021; Accepted August 5, 2021

DOI: $10.3892 / \mathrm{etm} .2021 .10997$

\begin{abstract}
Aggressive prolactinoma (APRL) is a subgroup of aggressive pituitary tumors (accounting for $10 \%$ of all hypophyseal neoplasia) which are defined by: invasion based on radiological and/or histological features, a higher proliferation profile when compared to typical adenomas and rapidly developing resistance to standard medication/protocols in addition to an increased risk of early recurrence. This is a narrative review focusing on APRL in terms of both presentation and management. Upon admission, the suggestive features may include increased serum prolactin with a large tumor diameter (mainly $>4 \mathrm{~cm}$ ), male sex, early age at diagnosis ( $<20$ years), and genetic predisposition [multiple
\end{abstract}

Correspondence to: Dr Mihai Cristian Dumitrascu, Department of Obstetrics and Gynecology, 'Carol Davila' University of Medicine and Pharmacy, 37 Dionisie Lupu Street, 020021 Bucharest, Romania E-mail: drdumitrascu@yahoo.com

Abbreviations: AIP, aryl hydrocarbon receptor-interacting protein; APRL, aggressive prolactinoma; PT, aggressive pituitary tumor; BRCA, breast cancer gene; DA, dopamine agonist; ESE, European Society of Endocrinology; EGFR, epidermal growth factor receptor; MEN1, multiple endocrine neoplasia type 1; MMP-9, matrix metalloproteinase 9; MGMT, O(6)-methylguanine-DNA methyltransferase; mTOR, mammalian target of rapamycin; PC, pituitary carcinoma; PTTG1, pituitary tumor transforming gene 1; TKI, tyrosine kinase inhibitor; VEGF, vascular endothelial growth factor

Key words: prolactinoma, aggressive prolactinoma, pituitary carcinoma, aggressive pituitary tumor, cabergoline, temozolomide, hypophysectomy, prolactin, menses, menstrual cycle endocrine neoplasia type 1 (MEN1), aryl hydrocarbon receptor interacting protein $(A I P)$, succinate dehydrogenase $(S D H x)$ gene mutations]. Potential prognostic factors are indicated by assessment of E-cadherin, matrix metalloproteinase (MMP)-9, and vascular endothelial growth factor (VEGF) status. Furthermore, during management, APRL may be associated with dopamine agonist (DA) resistance (described in 10-20\% of all prolactinomas), post-hypophysectomy relapse, mitotic count $>2, \mathrm{Ki}-67$ proliferation index $\geq 3 \%$, the need for radiotherapy, lack of response in terms of controlling prolactin levels and tumor growth despite multimodal therapy. However, none of these as an isolated element serves as a surrogate of APRL diagnosis. A fourth-line therapy is necessary with temozolomide, an oral alkylating chemotherapeutic agent, that may induce tumor reduction and serum prolactin reduction in $75 \%$ of cases but only $8 \%$ have a normalization of prolactin levels. Controversies surrounding the duration of therapy still exist; also regarding the fifth-line therapy, post-temozolomide intervention. Recent data suggest alternatives such as somatostatin analogues (pasireotide), checkpoint inhibitors (ipilimumab, nivolumab), tyrosine kinase inhibitors (TKIs) (lapatinib), and mTOR inhibitors (everolimus). APRL represents a complex condition that is still challenging, and multimodal therapy is essential.

\section{Contents}

1. Introduction

2. Aim of the review

3. Presentation

4. Management

5. Future considerations

6. Conclusions 


\section{Introduction}

Aggressive prolactinoma (APRL) is a subgroup of aggressive pituitary tumors (APTs) which represent a relatively new concept defined by three main features: tumor invasion based on radiological and/or histological features; a higher proliferation profile than usual pituitary adenomas (also called typical adenomas) and aggressive behavior such as rapidly developing resistance to standard medication/protocols and increased risk of early recurrence after surgical removal or after achieving transitory control under traditional medical therapy (1). APTs, representing around $10 \%$ of all pituitary tumors, require a complex multidisciplinary multimodal approach that may include: neurosurgery (numerous repeated procedures), radiotherapy, peptide receptor radionuclide therapy, combined medical therapy depending on secretory profile (such as cabergoline, pasireotide), but also uncommon medication as temozolomide (an alkylating agent) if standard regimes are inefficient (2).

Diagnosis of pituitary carcinoma (PC) is positive only if distant or cerebrospinal fluid metastases are identified (3). APTs and PC represents an atypical subcategory of pituitary tumors which otherwise usually display a more favorable profile, thus requiring a particular approach as pointed by 2018 ESE (European Society of Endocrinology) guideline (4).

Generally, prolactinoma is a subgroup of functioning pituitary adenomas (a category that also includes corticotropinoma, somatotropinoma, gonadotropinoma, and thyrotropinoma), knowing that the most frequent abnormal pituitary secretion is prolactin (between 47 and $66 \%$ of all secretor pituitary adenomas, depending on the studies) (5). Functioning and non-functioning hypophyseal adenomas represent $15 \%$ of all intra-cranial tumors, being considered one of the most frequent intra-cranial neoplasia (6). Their associated co-morbidities due to the tumor itself and due to hypersecretion/hyposecretion involve a relatively high rate of morbidity and mortality (7).

The clinical spectrum due to hyperprolactinemia of tumor origin varies from different manifestations of male/female hypogonadism, mammary anomalies such as galactorrhea up to osteoporosis and associated fractures and cardiovascular risk (8-10). In addition, prolactinomas of more than $1 \mathrm{~cm}$ in diameter up to giant prolactin-secreting tumors are associated with local mass effects as seen in other pituitary tumors, regardless of their secretor features (11). Some syndromic prolactinomas are synchronous or asynchronous with other non-pituitary tumors as seen in adrenal glands or parathyroids $(12,13)$.

A total of $95 \%$ of all pituitary adenomas are sporadic, while germline mutations have been reported, for instance, multiple endocrine neoplasia type 1 (MEN1), aryl hydrocarbon receptor-interacting protein $(A I P)$, and succinate dehydrogenase (SDHx) (14). AIP and MEN1 mutations are correlated with a diagnosis of patients younger than 30 years of age (or even 20 years); AIP mutation is mostly associated with gigantism and large tumors; Xq26.3 mutations are associated with pituitary tumors in very young children (15). Prolactinomas with underlying $A I P$ mutations represent a small subset of tumors with a familial pattern which otherwise are typically growth hormone-producing tumors or non-functioning adenomas (16). Both AIP- and MEN1-related prolactinomas may be large and usually therapy resistant, especially $A I P$-associated prolactinomas, as similarly seen in somatotropinomas $(17,18)$.

A complex multidisciplinary management may be require for pituitary adenomas since their first line of approach is surgical removal, except for prolactinomas which traditionally respond to medical therapy such as dopamine agonists (DAs) and also except for non-functioning microadenomas (usually named incidentalomas) that do not require a specific approach, only imaging follow-up (19-21).

\section{Aim of the review}

We aimed to focus on APRL in terms of both presentation, but also management and associated prognostic factors (Fig. 1). This is a narrative review based on a PubMed search using key words, 'aggressive prolactinoma', 'aggressive pituitary tumor', and 'pituitary carcinoma'. A number of 81 references are included from 2009 to 2021. Inclusion criteria included English language and full-length articles. Exclusion criteria included papers published before 2009.

\section{Presentation}

Prolactinoma affects both females and males, having an incidence of 3-5 cases/100,000 individuals/year and a general prevalence of 50 cases $/ 100,000$ individuals which is higher in subjects with hypogonadism since increased prolactin levels induce central inhibition of gonadotropes through kisspeptin neurons (22). The detection of APRLs may not start from the clinical presentation itself, but the imaging detection of a large pituitary mass in addition to large mass-associated symptoms and signs and extremely elevated prolactin levels are important clues, further followed by a poor response to DA medication and need for neurosurgery/early relapse after hypophysectomy due to the unusual rapid growth (23). Generally, a patient with prolactinoma becomes a neurosurgery candidate in the event apoplexy or cystic transformation is present, neurological/ophthalmic deficit has been identified, and the patient develops DA resistance or intolerance (24).

Post-operative histological and immunohistochemistry reports provide additional elements of the aggressive profile in association with clinical outcome after removal. This can sometimes occur from the beginning even when the surgical procedure is for debulking purposes only (25).

Additional poor prognostic elements are represented by epigenetic data regarding APTs that demonstrate a high DNA methyltransferase overexpression, p53-related histone anomalies, and upregulation of citrullinating enzymes. Yet, currently research involving new predictive factors of aggressive behavior is still in progress (26). Sometimes the term 'refractory' pituitary adenoma is used as an open category of APT with high Ki-67, rapid growth and lack of response to a traditional guideline approach (25).

Overall, the cluster of elements used for evaluating the potential aggressive behavior starts with high prolactin levels at first admission and large diameter upon computed tomography or magnetic resonance imaging; yet these themselves do not necessarily indicate an APRL $(27,28)$. If the patient is a male or there is an early age at diagnosis or a genetic predisposition is identified, the prognosis may be poor $(27,29)$. 
Suggestive elements:

- Extremely high serum prolactin

- Large size (>4 cm)

- Imaging with invasion profile

- Male sex

- Early age at diagnosis (<20 years)

- Genetic predisposition (MEM, MP, SDHx mutations)

- Histological report with invasive profile

- Mitotic count $>2$

- Ki-67 proliferation index $3 \%$

\section{Management:}

- Dopamine agonist resistance

- Need for neurosurgery

- Need for radiotherapy 4111

- Early post-operatory relapse

- Lack of serum prolactin control and tumor growth despite multimodal therapy

- IV-line therapy: temozolomide

Alternatives:

- Somatostatin analogues (pasireotide)

- Checkpoint inhibitors (ipilimumab, nivolumab)

- Tyrosine kinase inhibitors (lapatinib)

- mTOR inhibitors (everolimus)

Potential useful assessments:

- E-cadherin

- Matrix metalloproteinase 9

- Vascular endothelial growth factor

Figure 1. Approach to aggressive prolactinoma: from suggestive elements to management (based on refs. 1-79).

Furthermore, the development of DA (mostly cabergoline) resistance represents another useful clue $(27,30)$. Since the subject is referred to neurosurgery, the post-hypophysectomy histological report pointing out a mitotic count higher than 2 and a Ki-67 at least $3 \%$ is an indicator of increased proliferation risk. Yet, in the case that we analyze these characteristics apart from all other elements, these do not necessarily indicate an APRL (27,31). The evolution of prolactin levels and tumor size under multimodal therapy are also indicators of APRL $(27,32)$.

Other indicators of poor prognosis may be provided by the assessment of E-cadherin, matrix metalloproteinase (MMP)-9, growth factors such as vascular endothelial growth factor (VEGF), and abnormal gene expression (AIP, MEN1, $p 53)$ or even breast cancer gene 1 (BRCAI) mutation has been described (27,33). At first glance, no single element of the features mentioned above is enough to reveal an APRL. This is a step by step process, while the clear difference between an adenoma and a carcinoma comes only with confirmation of metastases, not by just analyzing the pituitary tumor histology and immunohistochemistry $(19,27)$.

\section{Management}

Management of APRLs goes beyond the typical scenario of most prolactinoma cases that are not candidate to non-medical approach (34). In this particular situation, a complex team is eventually expected to be necessary, including approaches in neurosurgery, radiotherapy, and even oncology since the APRL profile is contoured step by step starting with the identification of a large invasive tumor and continuing to small changes in serum prolactin levels under DA (35). A total of $1-5 \%$ of prolactinomas are giant at presentation (a diameter larger than $4 \mathrm{~cm}$ ), while most macro-prolactinomas have a diameter between 1 and $4 \mathrm{~cm} \mathrm{(36).} \mathrm{DA} \mathrm{is} \mathrm{expected} \mathrm{to} \mathrm{induce} \mathrm{tumor} \mathrm{shrinkage}$ and normalization of prolactin levels due to the abundant expression of type 2 dopamine receptors; yet $10-20 \%$ of prolactinomas are DA resistant (10\% for cabergoline, respective $20-30 \%$ for bromocriptine; thus bromocriptine-resistance patients should be first switched to cabergoline with an $80 \%$ expected rate of response in most cases of prolactinomas) (37). APRLs are frequently resistant to traditional therapy with DA (mainly cabergoline); DA resistance is also seen in familial forms prolactinomas with underlying AIP or MEN1 mutations (38). In patients younger than 20 years, DA resistance is correlated with high serum levels of prolactin at first presentation and increased tumor diameter in addition to AIP/MENI mutations which represent an independent predictor factor for the poor rate of DA response, accounting for $14 \%$ of all cases with suboptimal evolution under DA (39). DA resistance is an essential feature of APRLs, but, on the other hand, not all DA-resistant prolactinomas are actually APRLs $(40,41)$. According to current opinions, DA resistance means the lack of achieving normal prolactin levels and at least 30\% reduction in the maximum diameter under high cabergoline doses (at least $3.5 \mathrm{mg} /$ week) $(40,41)$. The most useful clinical element of response is restoration of gonadal axe function. Usually lifelong administration of cabergoline is necessary in responsive cases since stopping the medication is associated with a $60-80 \%$ relapse rate (42).

Subjects with DA resistance become candidates for hypophysectomy and some experts consider that early recognition of patients who are DA non-responsive improves the success of the neurosurgical procedure due to tumor-associated fibrosis which is a direct contributor to incomplete resection (43-45). Retrospective post-surgery studies have shown that a value of 
Ki-67 of more than $3 \%$ is associated with a higher risk of resistance to medication and post-operatory relapse; however, Ki-67 is not the only useful indicator and this isolated value itself is subject to controversy regarding APRL prognosis $(44,45)$. In cases with persistent or progressive disease after surgery or if only debulking surgery is feasible, DA should be re-considered in addition to irradiation therapy, preferable gamma knife (46).

Radiotherapy for pituitary tumors is useful if other medical and surgical procedures do not control the condition; the risk of hypopituitarism is high and in certain circumstances the risk of a secondary brain tumor should be taken into account (47). Some authors suggest that radiation therapy applied for invasive prolactinomas that are non-responsive to surgery and DA may be a promotor of distant metastases, thus subscribing to a PC profile, but this aspect is still a matter of debate (48).

Temozolomide, an active chemotherapy drug, represents a fourth-line therapy in prolactinomas after DA, transphenoidal selective hypophysectomy and radiotherapy (49). A specific time frame for its introduction into the patient regime varies, but prompt intervention is recommended (50). As pointed by the 2018 ESE guideline, temozolomide as a single medication is the first-line medical therapy after specific standard therapies have failed; 3 cycles are needed in order to decide if the patient is a responder, in which case therapy is prolonged for 6 months (4). However, following this article, controversies emerged at temozolomide withdrawn since an efficient fifth-line therapy (or second-line treatment) after temozolomide is still lacking and re-starting the same medication usually fails to achieve a relevant clinical and imaging response. Thus, the length of the therapy may go up to 12 cycles in many studies and even up to $14-59$ cycles if it is well tolerated (a cycle means a daily oral dose of $50-200 \mathrm{mg} / \mathrm{sqm}, 5$ out of 28 days) (51). Prolonged administration is under consideration in many cases despite guidelines based on an individual multidisciplinary decision (52).

Almalki et al described rates of response following temozolomide therapy as following: $76 \%$ of patients with tumor reduction, $75 \%$ with serum prolactin level reduction, $8 \%$ with normalization of prolactin, and a failure rate of $20.6 \%$ of subjects (53). It seems that APRLs had a higher rate of response to temozolomide than corticotropinomas $(60 \%)$ and somatotropinomas (26.7\%) (54). The assay of O(6)-methylguanine-DNA methyltransferase (MGMT) status may serve as a surrogate to anticipate the temozolomide response rate (50).

\section{Future considerations}

As mentioned, APRL is part of a syndromic combination similar to that seen in MEN1 syndrome, and in this particular circumstance the aggressive profile cannot be predicted by a specific gene mutation configuration itself but by a constellation of factors. However, the presence of other tumors such as associated neuroendocrine neoplasia of the pancreas or adrenals are direct contributors to a more severe overall prognosis $(55,56)$.

Once temozolomide is inefficient or it is not tolerated by a patient with APRLs, the therapeutic options are extremely limited (57). A few cases of prolactinomas, respective corticotropinomas with very aggressive profile have been recently reported as candidates for new drugs such as checkpoint inhibitors (ipilimumab, nivolumab) after temozolomide has failed to control the APT (58). The ErbB pathway is newly detected as correlated with aggressive profile of prolactinoma and DA resistance in APRL; thus, therapy with lapatinib, an oral tyrosine kinase inhibitor (TKI) targeting ErbB1-epidermal growth factor receptor (EGFR) is under evaluation, already being included in Phase 2 a clinical trials (59). Somatostatin analogue therapy for APRLs has demonstrated promising results, as well as its use in DA-resistant prolactinomas which are not APRLs (60-62). If a pituitary neuroendocrine tumor with prolactin production has somatostatin receptor (SSTR) expression, especially type 5 more than type 2, pasireotide long-acting release (LAR) (40 mg per month) may be beneficial $(61,62)$.

Of course, a mixed secretion of both prolactin and growth hormone is associated with a better response to somatostatin analogues (63). In APRLs, cystic transformation and tumor necrosis have been reported under pasireotide LAR (64). Pasireotide, outside the fact that it represents a second-line medical therapy in acromegaly, is also used in Cushing disease and some pancreatic neuroendocrine neoplasia $(65,66)$. In addition, isolated case reports have introduced pasireotide as an alternative to prolactinomas that are resistant to standard therapies, not necessarily APRLs (67). The use of SSTR immunostaining after neurosurgery may predict a potential response to this class of drugs, as currently being used in acromegaly treatment (68-70).

Moreover, estrogen modulators and metformin have been suggested for APRLs, but the findings still require statistical validation since the current level of evidence is low (60). The mammalian target of rapamycin (mTOR) inhibitor, everolimus, was applied as an off-label therapy in one case of APRL in combination with cabergoline and the results were encouraging (71). Generally, everolimus has been approved for pancreatic neuroendocrine neoplasia also in combination with somatostatin analogues including ocreotide or lanreotide $(72,73)$. The mTOR pathway is involved in the development of tumors derived from pituitary cells through intervention of the pituitary tumor transforming gene (PTTG1) (74). The observation is consistent for GH-secreting tumors as well (75). Murine experiments suggest that inhibition of the mTOR pathway may increase the cytotoxicity induced by temozolomide (76). The use of temozolomide for APTs dates since 2006, and it is generally considered to be well tolerated (the most common side effects are nausea, fatigue, different types of cytopenia) (77). It is usually recommended for treating brain cancers such as glioblastoma $(78,79)$.

\section{Conclusions}

APRLs represent a challenging condition that requires a multimodal approach; in addition to a standard three-line therapy, temozolomide medication is required.

\section{Acknowledgements}

Not applicable.

\section{Funding}

No funding was received. 


\section{Availability of data and materials}

Not applicable.

\section{Authors' contributions}

AV drafted the manuscript and critically revised the final form. FS researched the literature data, and AP critically revised the manuscript in light of the literature findings. MCD is the corresponding author and revised the literature findings. MC drafted the manuscript and reviewed the references. RCP researched the literature and AG approved the final form in light of the literature data. All authors read and approved the final manuscript for publication.

\section{Ethics approval and consent to participate}

Not applicable.

\section{Patient consent for publication}

Not applicable.

\section{Competing interests}

The authors declare that they have no competing interests.

\section{References}

1. Chatzellis E, Alexandraki KI, Androulakis II and Kaltsas G: Aggressive pituitary tumors. Neuroendocrinology 101: 87-104, 2015.

2. Iglesias P, Magallón R, Mitjavila M, Rodríguez Berrocal V, Pian H and Díez JJ: Multimodal therapy in aggressive pituitary tumors. Endocrinol Diabetes Nutr (Engl Ed) 67: 469-485, 2020.

3. Ilie MD, Jouanneau E and Raverot G: Aggressive pituitary adenomas and carcinomas. Endocrinol Metab Clin North Am 49 505-515, 2020.

4. Raverot G, Burman P, McCormack A, Heaney A, Petersenn S, Popovic V, Trouillas J and Dekkers OM; European Society of Endocrinology: European society of endocrinology clinical practice guidelines for the management of aggressive pituitary tumours and carcinomas. Eur J Endocrinol 178: G1-G24, 2018.

5. Thakkar K, Sarathi V and Shah NS: Current Status of diagnosis and management for functioning pituitary tumors: Part I. Neurol India 68 (Suppl 1): S13-S19, 2020.

6. Mehta GU and Lonser RR: Management of hormone-secreting pituitary adenomas. Neuro Oncol 19: 762-773, 2017.

7. Araujo-Castro M, Berrocal VR and Pascual-Corrales E: Pituitary tumors: Epidemiology and clinical presentation spectrum. Hormones (Athens) 19: 145-155, 2020.

8. Poiana C, Chirita C, Carsote M, Hortopan D and Goldstein A: Galactocele and prolactinoma-a pathogenic association? Maturitas 6: 98-102, 2009.

9. Samperi I, Lithgow K and Karavitaki N: Hyperprolactinaemia. J Clin Med 8: 2203, 2019.

10. Greenman Y: Prolactinomas and menopause: Any changes in management? Pituitary 23: 58-64, 2020.

11. Tirosh A and Shimon I: Management of macroprolactinomas. Clin Diabetes Endocrinol 1: 5, 2015.

12. Iliesiu A, Ungureanu IA, Petca A, Constantin MM, Petca RC, Sandru F, Constantin T and Dumitrascu MC: Paraganglioma presenting as a mesenteric cystic mass: A case report. Exp Ther Med 20: 2489-2492, 2020.

13. Nassiri F, Cusimano MD, Scheithauer BW, Rotondo F, Fazio A, Syro LV, Kovacs K and Lloyd RV: Prolactinomas: Diagnosis and treatment. Expert Rev Endocrinol Metab 7: 233-241, 2012.

14. Van den Broek MF, van Nesselrooij BP, Verrijn Stuart AA, van Leeuwaarde RS and Valk GD: Clinical relevance of genetic analysis in patients with pituitary adenomas: A systematic review. Front Endocrinol (Lausanne) 10: 837, 2019.
15. Barry S and Korbonits M: Update on the genetics of pituitary tumors. Endocrinol Metab Clin North Am 49: 433-452, 2020

16. Carty DM, Harte R, Drummond RS, Ward R, Magid K, Collier D, Owens $M$ and Korbonits M: AIP variant causing familial prolactinoma. Pituitary 24: 48-52, 2021.

17. Daly AF, Cano DA, Venegas-Moreno E, Petrossians P, Dios E, Castermans E, Flores-Martínez A, Bours V, Beckers A and Soto-Moreno A: AIP and MEN1 mutations and AIP immunohistochemistry in pituitary adenomas in a tertiary referral center. Endocr Connect 8: 338-348, 2019.

18. Schernthaner-Reiter MH, Trivellin G and Stratakis CA: Interaction of AIP with protein kinase A (cAMP-dependent protein kinase). Hum Mol Genet 27: 2604-2613, 2018.

19. Cuny T, Barlier A, Feelders R, Weryha G, Hofland LJ, Ferone D and Gatto F: Medical therapies in pituitary adenomas: Current rationale for the use and future perspectives. Ann Endocrinol (Paris) 76: 43-58, 2015.

20. Honegger J, Nasi-Kordhishti I, Aboutaha N and Giese S: Surgery for prolactinomas: A better choice? Pituitary 23: 45-51, 2020.

21. Kuo M, Maya MM, Bonert V and Melmed S: Prospective evaluation of incidental pituitary imaging findings in the Sella Turcica. J Endocr Soc 5: bvaa186, 2020.

22. Chanson P and Maiter D: The epidemiology, diagnosis and treatment of Prolactinomas: The old and the new. Best Pract Res Clin Endocrinol Metab 33: 101290, 2019.

23. Lasolle H, Ilie MD and Raverot G: Aggressive prolactinomas: How to manage? Pituitary 23: 70-77, 2020.

24. Donoho DA and Laws ER Jr: The role of surgery in the management of prolactinomas. Neurosurg Clin N Am 30: 509-514, 2019.

25. Dai C, Liu X, Ma W and Wang R: The treatment of refractory pituitary adenomas. Front Endocrinol (Lausanne) 10: 334, 2019.

26. Hauser BM, Lau A, Gupta S, Bi WL and Dunn IF: The Epigenomics of pituitary adenoma. Front Endocrinol (Lausanne) 10: 290,2019

27. Trouillas J, Delgrange E, Wierinckx A, Vasiljevic A, Jouanneau E, BurmanPandRaverotG: Clinical,pathological,andmolecularfactors of aggressiveness in lactotroph tumours. Neuroendocrinology 109: 70-76, 2019.

28. Vroonen L, Daly AF and Beckers A: Epidemiology and management challenges in prolactinomas. Neuroendocrinology 109: 20-27, 2019.

29. Yoo F, Chan C, Kuan EC, Bergsneider M and Wang MB: Comparison of male and female prolactinoma patients requiring surgical intervention. J Neurol Surg B Skull Base 79: 394-400, 2018.

30. Olarescu NC, Perez-Rivas LG, Gatto F, Cuny T, Tichomirowa MA, Tamagno G and Gahete MD; EYRC (ENEA Young Researcher Committee): Aggressive and malignant prolactinomas. Neuroendocrinology 109: 57-69, 2019.

31. Glezer A and Bronstein MD: Prolactinomas. Endocrinol Metab Clin North Am 44: 71-78, 2015.

32. Langlois F, McCartney S and Fleseriu M: Recent progress in the medical therapy of pituitary tumors. Endocrinol Metab (Seoul) 32: 162-170, 2017

33. Bettencourt-Silva R, Queirós J, Pereira J and Carvalho D: Giant prolactinoma, germline BRCA1 mutation, and depression: A case report. J Med Case Rep 12: 360, 2018.

34. Huynh PP, Ishii LE and Ishii M: Prolactinomas. JAMA 325: 195 , 2021.

35. Giraldi EA and Ioachimescu AG: The role of dopamine agonists in pituitary adenomas. Endocrinol Metab Clin North Am 49: 453-474, 2020.

36. Shimon I: Giant prolactinomas. Neuroendocrinology 109: 51-56, 2019.

37. Sahakian N, Castinetti F, Dufour H, Graillon T, Romanet $P$, Barlier A, Brue T and Cuny T: Clinical management of difficult to treat macroprolactinomas. Expert Rev Endocrinol Metab 14: 179-192, 2019.

38. Vroonen L, Daly AF and Beckers A: Challenges and controversies in the treatment of prolactinomas. Expert Rev Endocrinol Metab 9: 593-604, 2014.

39. Salenave S, Ancelle D, Bahougne T, Raverot G, Kamenický P, Bouligand J, Guiochon-Mantel A, Linglart A, Souchon PF, Nicolino M, et al: Macroprolactinomas in children and adolescents: Factors associated with the response to treatment in 77 patients. J Clin Endocrinol Metab 100: 1177-1186, 2015.

40. Vermeulen E, D'Haens J, Stadnik T, Unuane D, Barbe K, Van Velthoven V and Gläsker S: Predictors of dopamine agonist resistance in prolactinoma patients. BMC Endocr Disord 20: 68, 2020 . 
41. Maiter D: Management of dopamine agonist-resistant prolactinoma. Neuroendocrinology 109: 42-50, 2019.

42. Espinosa-Cárdenas E, Sánchez-García M, Ramírez-Rentería C, Mendoza-Zubieta V, Sosa-Eroza E and Mercado M: High biochemical recurrence rate after withdrawal of cabergoline in prolactinomas: Is it necessary to restart treatment? Endocrine 70: 143-149, 2020

43. Vermeulen E, D'Haens J, Stadnik T, Unuane D, Barbe K, Van Velthoven V and Gläsker S: Predictors of dopamine agonist resistance in prolactinoma patients. BMC Endocr Disord 20: 68, 2020.

44. Lu C, Liu Y, Lu Z and Huan C: Ki-67 and clinical correlations in patients with resistant prolactinomas. Ann Clin Lab Sci 50 199-204, 2020

45. Stiles CE, Steeds RP and Drake WM: Monitoring patients receiving dopamine agonist therapy for hyperprolactinaemia. Ann Endocrinol (Paris) 82: 182-186, 2021.

46. Souteiro P, Belo S and Carvalho D: Dopamine agonists in prolactinomas: When to withdraw? Pituitary 23: 38-44, 2020.

47. Castinetti F: Radiation techniques in aggressive pituitary tumours and carcinomas. Rev Endocr Metab Disord 21: 287-292, 2020.

48. Pérez Pinzón J, González-Devia D, Kattah Calderón W, López Panqueva RDP and Jiménez Hakim E: Unusual course of an aggressive pituitary prolactinoma: Case report and review of the literature. Case Rep Neurol 11: 148-156, 2019.

49. Barkhoudarian G, Palejwala SK, Ogunbameru R, Wei H, Eisenberg A and Kelly DF: Early recognition and initiation of temozolomide chemotherapy for refractory, invasive pituitary macroprolactinoma with long-term sustained remission. World Neurosurg 118: 118-124, 2018.

50. Chen C, Yin S, Zhang S, Wang M, Hu Y, Zhou P and Jiang S: Treatment of aggressive prolactinoma with temozolomide: A case report and review of literature up to date. Medicine (Baltimore) 96: e8733, 2017.

51. Lizzul L, Lombardi G, Barbot M, Ceccato F, Gardiman MP Regazzo D, Bellu L, Mazza E, Losa M and Scaroni C: Longcourse temozolomide in aggressive pituitary adenoma: Real-life experience in two tertiary care centers and review of the literature. Pituitary 23: 359-366, 2020.

52. Elbelt U, Schlaffer SM, Buchfelder M, Knappe UJ Vila G, Micko A, Deutschbein T, Unger N, Lammert A, Topuzoglu-Müller T, et al: Efficacy of temozolomide therapy in patients with aggressive pituitary adenomas and carcinomas-a German survey. J Clin Endocrinol Metab 105: dgz211, 2020.

53. Almalki MH, Aljoaib NN, Alotaibi MJ, Aldabas BS, Wahedi TS, Ahmad MM and Alshahrani F: Temozolomide therapy for resistant prolactin-secreting pituitary adenomas and carcinomas: A systematic review. Hormones (Athens) 16: 139-149, 2017.

54. Aydoğan Bİ, Ünlütürk U, Emral R and Güllü S: Course of aggressive somatotroph, corticotroph and mammotroph tumors under temozolomide; Report of three cases and review of the literature. Turk Neurosurg: May 7, 2017 (Epub ahead of print).

55. Mele C, Mencarelli M, Caputo M, Mai S, Pagano L, Aimaretti G, Scacchi M, Falchetti A, and Marzullo P: Phenotypes associated with MEN1 syndrome: A focus on genotype-phenotype correlations. Front Endocrinol (Lausanne) 11: 591501, 2020.

56. Miulescu R, Balaban DV, Sandru F and Jinga M: Cutaneous manifestations in pancreatic diseases-A review. J Clin Med 9: 2611,2020

57. Ortiz LD, Syro LV, Scheithauer BW, Rotondo F, Uribe H, Fadul CE, Horvath E and Kovacs K: Temozolomide in aggressive pituitary adenomas and carcinomas. Clinics (Sao Paulo) 67 (Suppl 1): S119-S123, 2012.

58. Duhamel C, Ilie MD, Salle H, Nassouri AS, Gaillard S, Deluche E, Assaker R, Mortier L, Cortet C and Raverot G: Immunotherapy in corticotroph and lactotroph aggressive tumors and carcinomas: Two case reports and a review of the literature. J Pers Med 10: 88, 2020.

59. Cooper O, Bonert VS, Rudnick J, Pressman BD, Lo J, Salvatori R, Yuen KCJ, Fleseriu M and Melmed S: EGFR/ErbB2-targeting lapatinib therapy for aggressive prolactinomas. J Clin Endocrinol Metab 106: e917-e925, 2021.
60. Souteiro P and Karavitaki N: Dopamine agonist resistant prolactinomas: Any alternative medical treatment? Pituitary 23: 27-37, 2020.

61. Raverot G, Vasiljevic A, Jouanneau E and Lasolle H: Confirmation of a new therapeutic option for aggressive or dopamine agonistresistant prolactin pituitary neuroendocrine tumors. Eur J Endocrinol 181: C1-C3, 2019.

62. Gomes-Porras M, Cárdenas-Salas J and Álvarez-Escolá C: Somatostatin analogs in clinical practice: A review. Int J Mol Sci 21: 1682, 2020.

63. Lv L, Jiang Y, Yin S, Hu Y, Chen C, Ma W, Jiang S and Zhou P: Mammosomatotroph and mixed somatotroph-lactotroph adenoma in acromegaly: A retrospective study with long-term follow-up. Endocrine 66: 310-318, 2019.

64. Coopmans EC, van Meyel SWF, Pieterman KJ, van Ipenburg JA Hofland LJ, Donga E, Daly AF, Beckers A, van der Lely AJ and Neggers SJCMM: Excellent response to pasireotide therapy in an aggressive and dopamine-resistant prolactinoma. Eur J Endocrinol 181: K21-K27, 2019.

65. Siddiqui M, Vora A, Ali S, Abramowitz J and Mirfakhraee S: Pasireotide: A novel treatment for tumor-induced hypoglycemia due to insulinoma and non-islet cell tumor hypoglycemia. J Endocr Soc 5: bvaa171, 2020.

66. Zhao N, Yang X, Li C, Ma J and Yin X: Efficacy and safety of pasireotide for Cushing's disease: A protocol for systematic review and meta-analysis. Medicine (Baltimore) 99: e23824, 2020.

67. Lasolle H, Vasiljevic A, Borson-Chazot F and Raverot G: Pasireotide: A potential therapeutic alternative for resistant prolactinoma. Ann Endocrinol (Paris) 80: 84-88, 2019.

68. Oki Y: Medical management of functioning pituitary adenoma: An update. Neurol Med Chir (Tokyo) 54: 958-965, 2014.

69. Valea A, Ghervan C, Carsote M, Morar A, Iacob I, Tomesc F, Pop DD and Georgescu C: Effects of combination therapy: Somatostatin analogues and dopamine agonists on GH and IGF1 levels in acromegaly. Clujul Med 88: 310-313, 2015.

70. Valea A, Carsote M, Ghervan C and Georgescu C: Glycemic profile in patients with acromegaly treated with somatostatin analogue. J Med Life 8: 82-86, 2015.

71. Zhang D, Way JS, Zhang X, Sergey M, Bergsneider M, Wang MB, Yong WH and Heaney AP: Effect of everolimus in treatment of aggressive prolactin-secreting pituitary adenomas. J Clin Endocrinol Metab 104: 1929-1936, 2019.

72. Zhang J, Francois R, Iyer R, Seshadri M, Zajac-Kaye M and Hochwald SN: Current understanding of the molecular biology of pancreatic neuroendocrine tumors. J Natl Cancer Inst 105: 1005-1017, 2013.

73. Wolin EM: The expanding role of somatostatin analogs in the management of neuroendocrine tumors. Gastrointest Cancer Res 5: 161-168, 2012

74. Chen R, Duan J, Li L, Ma Q, Sun Q, Ma J, Li C, Zhou X, Chen H, Jing Y, et al: mTOR promotes pituitary tumor development through activation of PTTG1. Oncogene 36: 979-988, 2017.

75. Gorshtein A, Rubinfeld H, Kendler E, Theodoropoulou M, Cerovac V, Stalla GK, Cohen ZR, Hadani M and Shimon I: Mammalian target of rapamycin inhibitors rapamycin and RAD001 (everolimus) induce anti-proliferative effects in GH-secreting pituitary tumor cells in vitro. Endocr Relat Cancer 16: 1017-1027, 2009.

76. Dai C, Zhang B, Liu X, Ma S, Yang Y, Yao Y, Feng M, Bao X, Li G, Wang J, et al: Inhibition of PI3K/AKT/mTOR pathway enhances temozolomide-induced cytotoxicity in pituitary adenoma cell lines in vitro and xenografted pituitary adenoma in female nude mice. Endocrinology 154: 1247-1259, 2013.

77. Whitelaw BC: How and when to use temozolomide to treat aggressive pituitary tumours. Endocr Relat Cancer 26: R545-R552, 2019.

78. Schreck KC and Grossman SA: Role of temozolomide in the treatment of cancers involving the central nervous system. Oncology (Williston Park) 32: 555-560, 2018.

79. Arora A and Somasundaram K: Glioblastoma vs temozolomide: Can the red queen race be won? Cancer Biol Ther 20: 1083-1090, 2019. 